\title{
Vibration Control Using Multilayer Piezoelectric Actuators: Towards Chatter Supression in Turning Operations ${ }^{1}$
}

\author{
Giuliana Sardi Venter ${ }^{a, *}$ (D), Maíra Martins da Silva ${ }^{b}$ \\ ${ }^{a}$ Universidade Federal do Paraná, Departamento de Engenharia Mecânica, Setor Tecnológico, \\ Av. Cel. Francisco H. dos Santos, 210, Curitiba, PR, Brasil \\ ${ }^{b}$ Universidade de São Paulo, Escola de Engenharia de São Carlos, Departamento de Engenharia \\ Mecânica, Av. Trabalhador São Carlense, 400, São Carlos, SP, Brasil
}

Received: February 19, 2020; Revised: May 27, 2020; Accepted: June 25, 2020

\begin{abstract}
Chatter causes poor surface quality and damage to tools in machining. Therefore, strategies for chatter monitoring and reduction are constantly under investigation. Among these strategies, the use of piezoelectric layers embedded in the tool-holder as sensors/actuators for control strategies had been proposed for improving the stability limit reducing the system vibration. Nevertheless, actuators implemented using a single piezoelectric layer may fail to suppress chatter due to its limited actuation power. A method for assembling a series multilayer piezoelectric actuator is then proposed, which can provide a better performance and ultimately suppress chatter in turning operations. This actuator is used with passive and active control and the system response is investigated using frequency response functions. Two fixation conditions are tested to analyze the robustness of the control strategies. The results show that the use of a multilayer piezoelectric actuator made it possible for a greater reduction in vibration amplitude in the chatter frequency.
\end{abstract}

Keywords: Internal turning, Passive Control, Active Control, Stability Limit.

\section{Introduction}

Chatter is a phenomenon that can arise under certain machining conditions, causing severe vibrations that lead to poor surface quality, excessive tool wear, and the necessity of rework ${ }^{1,2}$. This vibration occurs due to the complex interactions between the machine tool, the tool-holder, and the workpiece that involve interactions due to friction, thermodynamics, nonlinear forces, and chip modulation ${ }^{3}$. Given its prevalence of internal turning operations due to slender tool-holders, strategies to predict ${ }^{4,5}$ and control chatter $^{6-8}$ are paramount.

Chip thickness regeneration, caused by modulations left on the machined surface from previous rotations, is the main cause of chatter ${ }^{9-12}$. Machining parameters can influence these modulations, affecting closed-loop damping. A careful selection of machining parameters such as depth of cut and spindle speed can ensure that the system is under the stability limit, providing a stable cut. The stability limit can be represented on Stability Lobe Diagrams ${ }^{9}$, which provide visual identification of stable and unstable regions of machining parameters. Stability Lobe Diagrams are applied to characterize the system and may be used to predict chatter, as well as an aid to monitor and control it. There are several ways to numerically and experimentally estimate the Stability Lobe Diagrams, such as the ones presented by Ganguli et al. ${ }^{4}$, Urbikain et al. ${ }^{13}$ and Liu et al. ${ }^{14}$.

${ }^{1}$ This paper was presented in the 10th Brazilian Congress on Manufacturing Engineering, August 2019, São Carlos / SP

*email: giuliana.venter@ufpr.br
Selecting the right parameters may ensure a stable cut, however it hinders the overall productivity, as it guarantees a conservative selection. In order to improve the productivity and still provide a stable cut, strategies to increase the stability limit need to be used. It is well established that the structural damping has a strong correlation with the stability limit ${ }^{4,9-15}$. As one increases the system damping, the stability limit is raised, enabling a stable cut for a wider range of cutting parameters without the negative impact on productivity that a conservative choice of parameters may have.

System damping can be increased through passive or active control strategies. Passive strategies can use tuned mass dampers ${ }^{16}$, shunt strategies ${ }^{15,17}$, or other tool-holder modifications, such as the inclusion of spheres in a cavity in the center of the tool-holder ${ }^{18}$. Passive shunts act in the same way as tuned mass dampers ${ }^{19}$, but they offer advantages because they are compact and simple to manufacture. Inductive-resistive shunts can be electromechanically coupled to the tool-holder structure using piezoelectric material ${ }^{17}$. The use of velocity feedback control is an active control strategy that also increases the system damping ${ }^{12,17,20}$, as it actuates as virtual damping and can be implemented using the same piezoelectric actuator. Active strategies that require major tool-holder changes, such as using a variable stiffness vibration absorber, proposed by Liu et al. ${ }^{14}$ have the disadvantage of limiting the use of the tool-holder. By using piezoelectric actuators coupled to the tool-holder, one can choose to use different passive and active controls, generating a more versatile tool to control machining vibration. 
However, one possible drawback of using piezoelectric actuators is the control output limitation that is imposed by the available electrical tension ${ }^{21}$. The use of simple piezoelectric actuators, which are composed of a single layer of piezoelectric material, may not have the mechanical capability to suppress chatter's severe vibration due to such limitation $^{22}$. Often the electrical tension required is deterrent to the full mitigation of chatter. Thus, this research proposes a thorough method to manufacture a multilayer piezoelectric actuator, providing a higher actuation power, as the actuator is now composed of several piezoelectric layers. Some insights on this proposal have been described in Venter et al. ${ }^{20}$ This actuator is used to ascertain the vibration reduction capability of active control via velocity feedback control and passive control via inductive-resistive shunts. The system response is evaluated using experimentally obtained driving tip Frequency Response Functions (FRF), which were then fitted to a four-degree-of-fredom model using a polynomial multi-mode system identification that showed the increase in the damping ratio, which in turn implies in a higher stability limit. Two fixation conditions are tested to analyze the robustness and the potential to reduce the vibration amplitude for passive and active control strategies under different boundary conditions.

\section{The Relation Between Damping and the Stability Limit}

In this section, a simplified model of the dynamics during the turning process is derived using a single-degree-of-freedom model. The objective of this description is to show the relationship between the stability limit, $K_{\text {lim }}$, and the system damping. The stability limit is a physical property that varies according to the spindle velocity and determines the upper limit for the relation $K_{f} a$ for performing a stable cut ( $K_{\text {lim }} \geq K_{f} a$ ), where $a$ is the width-of-cut and $K_{f}$ is the cutting coefficient.

Figure 1 shows the tool and the workpiece during two consecutive cuts under a turning operation. In this figure, $w(t)$ and $w(t-T)$ are the cut waves at these two consecutive cuts, $T$ is the spindle revolution period and $h_{0}$ is the uncut chip thickness. The instantaneous chip thickness $h(t)$ can be described as:

$h(t)=h_{0}+w(t-T)-w(t)$

The cutting force $F_{c}$ can be approximated by a proportional value of the front area of the chip, $F_{c}(t)=K_{f} a h=K_{f} a\left(h_{0}+w(t-T)-w(t)\right)$. Considering that the tool-holder can be represented by a single degree-of-freedom system, its transfer function in the Laplace domain can be written as:

$$
G(s)=\frac{W(s)}{F C(s)}=\frac{1}{m s^{2}+c s+k}
$$

where $W(s)$ is the Laplace transformation of the cut wave $w(t), F C(s)$ is the Laplace transformation of the cutting force $F_{c}(t), m$ is the equivalent mass of the system, $c$ is the equivalent damping and $k$ is the equivalent stiffness. In the Laplace domain, the relation between the instantaneous

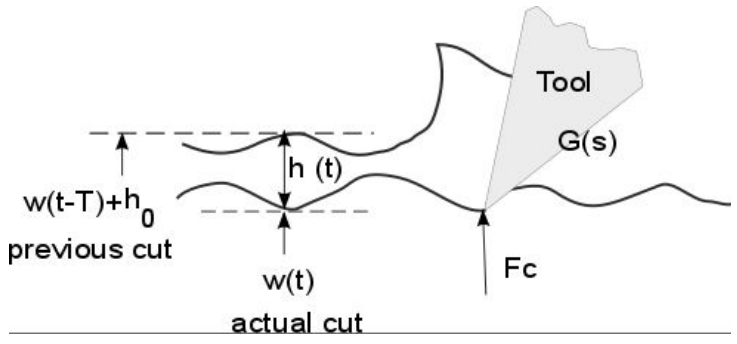

Figure 1. Two consecutive cuts, adapted from (4).

and the uncut chip thicknesses can be described as proposed by Tobias and Gurney ${ }^{9}$, Tlusty and Polocek ${ }^{10}$ and Merrit $^{11}$ :

$$
\frac{H}{H_{0}}(s)=\frac{1}{1+K_{f} a G(s)\left(1-e^{-s T}\right)}
$$

where $H(s)$ is the Laplace transformation of the instantaneous chip thickness $h(t)$ and $H_{0}(s)$ is the Laplace transformation of the uncut chip thickness $h_{0}$.

The stability limit $K_{\text {lim }}$ can be derived by evaluating the characteristic equation of the closed-loop system $1+K_{\text {lim }} G(s)\left(1-e^{-s T}\right)=0$ at the chatter frequency $\omega_{c}$, which may represent the limit of the stability. Since $K_{l i m}$ is the upper bound of a physical property, it should be real. Substituting $s=i \omega_{c}$ in the characteristic equation and using the Euler identity, $e^{-i \omega_{c} T}=\cos \left(\omega_{c} T\right)-i \operatorname{sen}\left(\omega_{c} T\right)$, the stability limit can be derived:

$$
\begin{aligned}
& K_{\text {lim }}=\frac{\Lambda_{R}\left(1-\cos \left(\omega_{c} T\right)\right)-\Lambda_{I} \operatorname{sen}\left(\omega_{c} T\right)}{2\left(\Lambda_{R}{ }^{2}+\Lambda_{I}^{2}\right)\left(1-\cos \left(\omega_{c} T\right)\right)}- \\
& \frac{\Lambda_{I}\left(1-\cos \left(\omega_{c} T\right)\right)+\Lambda_{R} \operatorname{sen}\left(\omega_{c} T\right)}{2\left(\Lambda_{R}{ }^{2}+\Lambda_{I}{ }^{2}\right)\left(1-\cos \left(\omega_{c} T\right)\right)} i
\end{aligned}
$$

in which $\Lambda_{R}=\operatorname{Re}\left[G\left(i \omega_{c}\right)\right]$ and $\Lambda_{I}=\operatorname{Imag}\left[G\left(i \omega_{c}\right)\right]$. The imaginary part of $K_{\text {lim }}$ should be null yielding the relations for calculating the chatter frequency $\omega_{c}$ and the stability limit $K_{\text {lim }}$ :

$$
\begin{aligned}
& \omega_{c} T=2 p \pi-2 \tan ^{-1}\left(\frac{\Lambda_{R}}{\Lambda_{I}}\right) \\
& K_{\text {lim }}=-\frac{1}{2 \Lambda_{R}}
\end{aligned}
$$

in which $p=1,2,3, \ldots$ Stability Lobe Diagrams can be generated plotting $K_{\text {lim }}$ versus the spindle velocity. Since $K_{\text {lim }}$ is the upper limit of a positive physical property ( $K_{\text {lim }} \geq K_{f} a$ ), the real part of the system transfer function, $\Lambda_{R}=\operatorname{Re}\left[G\left(i \omega_{c}\right)\right]=\left(k-m \omega_{c}\right) /\left(\left(k-m \omega_{c}\right)^{2}+\left(c \omega_{c}\right)^{2}\right)$ is a negative number according to Equation 6 . Therefore, $K_{\text {lim }}$ can be increased by reducing the module of the real part of the system transfer function. This can be accomplished by increasing the system damping ${ }^{4}$. In this manuscript, the damping is increased by the use of multilayer piezoelectric devices as illustrated in Figure 2. Figure 2a shows the exploitation of a passive strategy for increasing damping. In the passive configuration, the piezoelectric devices are connected to a 
shunt circuit $(Z)$ responsible for dissipating energy. Figure $2 b$ shows the exploitation of an active strategy for increasing damping. In the active configuration, the piezoelectric devices are connected to a control scheme responsible for actively reducing the system damping.

\section{Materials and Methods}

A multilayer piezoelectric actuator may be connected in series or parallel. The parallel connection should be used in the case of limited electrical tension outputs, as all patches receive the same electrical tension, and with larger inserts, which inherently require larger electrical tensions to produce mechanical deformation. In the case of a parallel connection, two or more piezoelectric patches receive the same electrical tension, which can be the maximum available tension. Besides, the capacitance of the new actuator is higher, reducing the optimal inductance used in the passive control strategies ${ }^{23}$. To obtain this connection, however, one must assert that there is an ideal insulation between the piezoelectric layers (see Figure 3), otherwise the actuator will malfunction. That may pose a challenge not only due to the necessity of ideal insulation, but also as the insulation layer may interfere with the expected electromechanical properties of the finished actuator.

To minimize such possible problems and in an experimental setup in which there is enough electrical tension available, a multilayer piezoelectric actuator in series is recommended. In order to produce the multilayer piezoelectric actuator in series it is necessary to use a substrate that will bond the piezoelectric inserts at the same time that it provides the electrical conductivity between the faces of the two inserts being connected, as can be seen in Figure 4. In this way, the piezoelectric insert terminals are connected in series and an electrical tension is applied to the terminals at the (a)

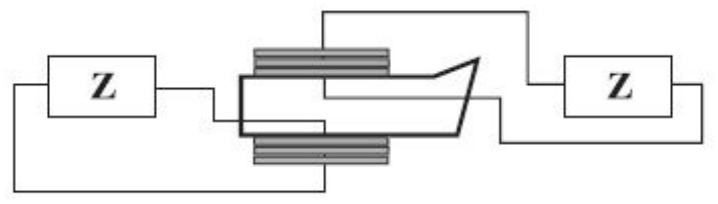

(b)

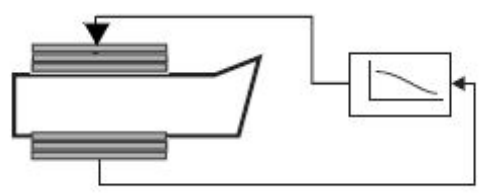

Figure 2. Multilayer piezoelectric layers: (a) passive strategy and (b) active strategy, adapted from (12).

(a)

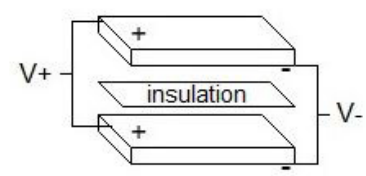

(b)

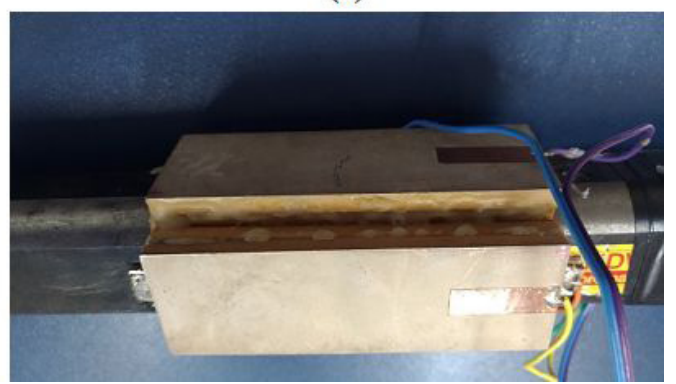

Figure 3. Assembly of multilayer piezoelectric actuator in parallel a) diagram; b) assembly with adhesive and silicon isolation layer. (b)

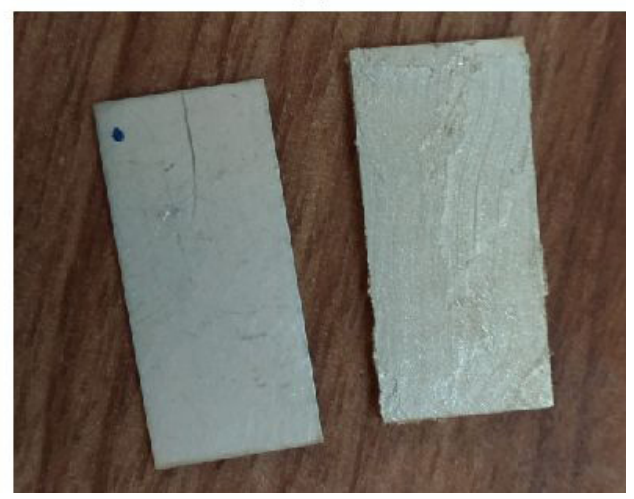

Figure 4. Assembly of multilayer piezoelectric actuators in series a) diagram; b) assembly with conductor adhesive. 
end of the actuator, producing an electrical tension on each patch that is the total tension divided by the number of piezoelectric patches.

Therefore, a multilayer piezoelectric actuator in series was produced. Two faces of a SANDVIK Coromant's A16T-SCLCR 4 tool-holder have been slightly modified in the direction of the feed to allow the piezoelectric pathes fixation (see Figure 5.a)). Three piezoelectric inserts were used to produce the finished multilayer actuator on each of the four faces of the tool-holder. Each PZT-5A piezoelectric insert had dimensions of $30 \mathrm{~mm} \times 14 \mathrm{~mm} \times 1 \mathrm{~mm}$.

With a thin layer of MG Chemicals Model 8331 silver-based conductive epoxy, the three piezoelectric inserts were bonded in series to obtain a multilayer piezoelectric actuator. Special care must be taken with the conductive epoxy so that it does not connect the two terminals of each piezoelectric insert through the side of the insert. Any trace of conductive epoxy that goes to the side of the tablet should be sanded to preserve the circuit's series connection. The cure period for each assembly was $24 \mathrm{~h}$, as defined by the manufacturer.

After the conductive epoxy curing, the actuators were coupled to the tool-holder faces with 3M Scotch-Weld DP460 epoxy adhesive. Copper tapes were used for electrical coupling. This step should be performed one face at a time, respecting the epoxy's $24 \mathrm{~h}$ cure period. Lastly, the electrical terminals were soldered to the previously applied copper strips. The finished tool-holder, which contains four multilayer actuators, one in each face, can be seen in Figure 5b).

After fixating the series multilayer piezoelectric actuators on the tool-holder, their capacitance was measured. In the depth-of-cut direction ( $y$-direction), the two inserts (one in each face of the tool-holder) have capacities $\mathrm{C}_{\mathrm{yl}}=2.14 \mathrm{nF}$ and $\mathrm{C}_{\mathrm{y} 2}=2.10 \mathrm{nF}$, which can total $\mathrm{a} \mathrm{C}_{\mathrm{y}}=4.24 \mathrm{nF}$ when both are connected in parallel to the amplifier, so they receive the same control signal, but in the opposite direction. In that case, you effectively produce one actuator that consists of two multilayer piezoelectric actuators in series, connected in parallel. In the direction of the feed ( $x$-direction), the two inserts (one in each face of the tool-holder) have capacities $\mathrm{C}_{\mathrm{x} 1}=2.08 \mathrm{nF}$ and $\mathrm{C}_{\mathrm{x} 2}=2.06 \mathrm{nF}$ which can total $\mathrm{a} \mathrm{C}_{\mathrm{x}}=4.14 \mathrm{nF}$ when both are connected in parallel to the amplifier so as to receive the same control signal, but in the opposite direction.

For the experiments performed in the laboratory, the tool-holder was fixated in an experimental setup, using a concrete block as a base, metal plates for positioning the tool-holder and screws for fixing. This condition simulates the lathe fixation that is the actual condition used in machining. These experiments were performed in the laboratory on only one direction as a proof of concept. However, as the tool-holder is intended to be used in internal turning, with a cutting force that inherently acts in both $\mathrm{x}$ and $\mathrm{y}$ directions, causing vibration, the control must be also performed in both directions. Such setup can be seen in Figure 6, adapted from Venter et $\mathrm{al}^{22}$.

As the condition of fixation may vary during the use life of the tool-holder, due to user error, different machines used and even variations on temperature and humidity, the dynamic properties of the tool-holder may vary as well. To test the robustness of the strategy under such different conditions, experiments were performed under two different fixation configurations. In the first configuration, the tool-holder was carefully fixed, to ensure alignment with the reference coordinate system in all directions. In the second configuration the tool-holder was intentionally misaligned with respect to the reference system during the clamping process, changing its natural frequency, as can be seen in Figure $6 a$ and $b$ ).

To obtain the frequency response functions, a power input to the system that spans a large frequency range is required, which is obtained through a chirp function, generated in a signal processor and sent to a shaker. Using the Data Physics

(a)

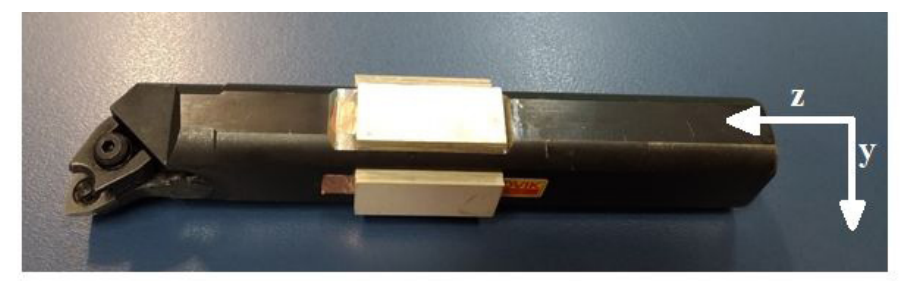

(b)

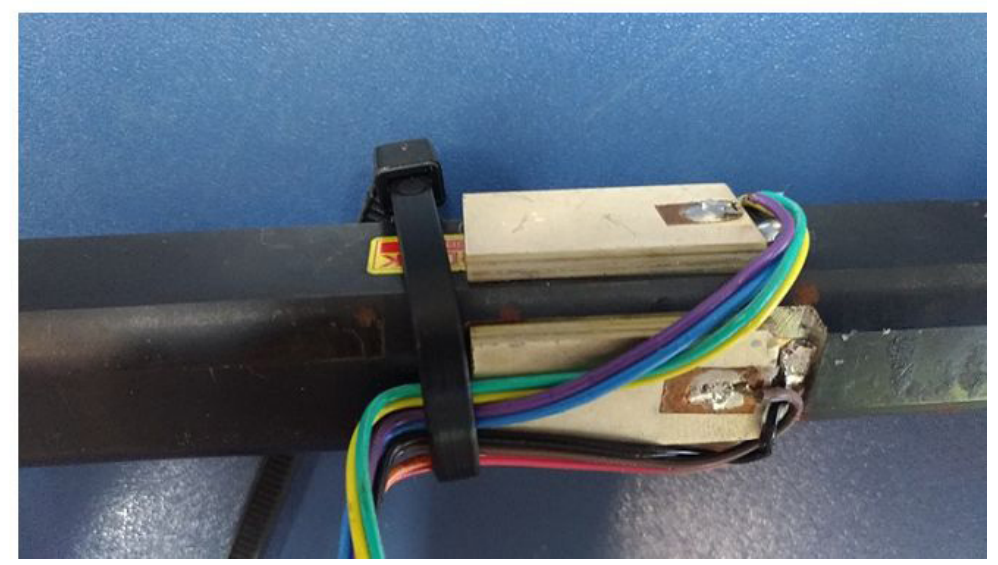

Figure 5. Assembly of multilayer piezoelectric actuators a) modified tool-holder; b) finished tool-holder with actuators. 
(a)

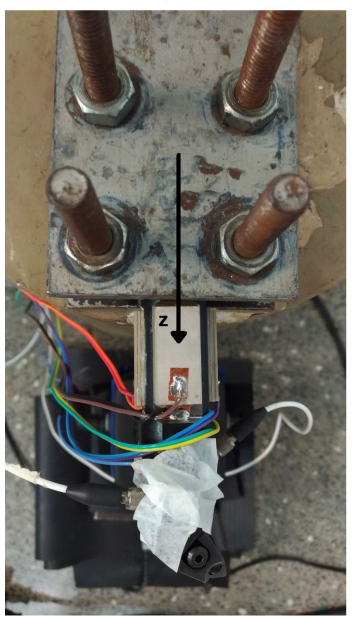

(b)

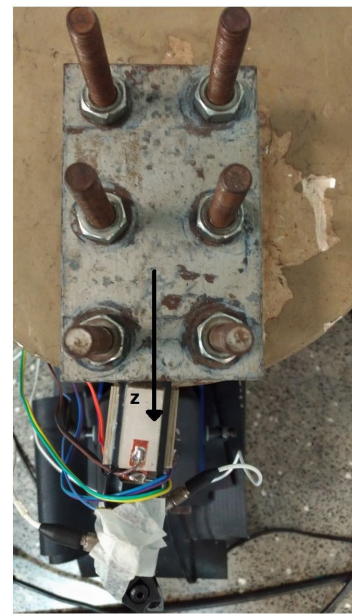

(c)

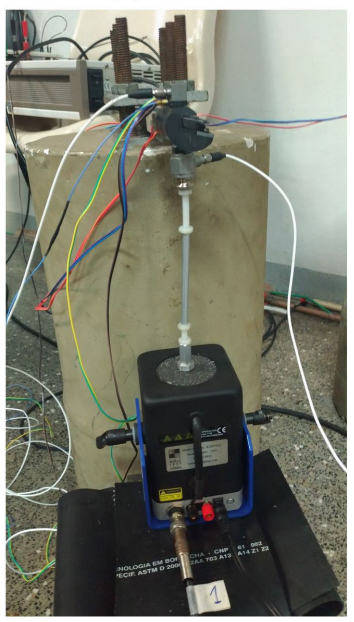

Figure 6. Experimental setup: a) first configuration - top view; b) second configuration - top view; c) lateral view.

Quattro FFT data acquisition system, a linear chirp signal from $50 \mathrm{~Hz}$ to $1250 \mathrm{~Hz}$ is generated. The shaker force is used as the input of the driving tip FRF, the output is the accelerometer positioned above the stinger, which measures the acceleration in the same direction as the force applied by the shaker. TMS Miniature SmartShakerTM K2004E01 shaker, Piezotronics PCB force transducer model 208C01 and Piezotronics PCB accelerometer model 352C65 were used. This experimental setup can be seen in Figure 6c). The FRF is approximated using the $\mathrm{H} 2$ approximation.

For the signal acquisition in the active control, the dSPACE ds1103 acquisition board was used. Since the board's I/O inputs do not support the ICP output from accelerometers, an ICP Model 482C SERIES signal conditioner from PCB Piezotronics was used. The signal was then acquired by dSPACE and introduced to Simulink, where acceleration signal processing and control signal generation occurs. This experimental scheme can be seen in Figure 7.

The acquired acceleration signal is treated with the sensitivity of the sensor, then passes through a high pass filter, with a $60 \mathrm{~Hz}$ cutoff frequency, in order to reduce experimental noises. After signal processing, the acceleration signal is integrated using a discrete real-time integrator with the Foward Euler method with integration steps at the same frequency as the $10^{-4} \mathrm{~s}$ signal acquisition sample rate (see Figure 8 ). The velocity can be understood as the integrated measured acceleration, which in turn represents the first derivative of the cut wave in the turning process. At this speed a $\mathrm{K}$ gain is applied which is set directly in the ControlDesk program. The generated control signal is applied to the piezoelectric actuator faces through the dSPACE plate.

To perform experiments in which passive control is used, the inductive-resistive circuit is directly connected to the piezoelectric actuators terminals in the $x$-direction using a protoboard. This is the same direction as the force applied by the shaker. This experimental scheme can be seen in Figure 7 b) and Figure 8.

In the passive control, the two $x$-direction piezoelectric actuators were used, which is the direction excited by the shaker. The two actuators, when connected in parallel to the protoboard, have a combined capacitance of $\mathrm{C}_{\mathrm{x}}=4.14 \mathrm{nF}$. Using the strategy of Hagood and von Flotow ${ }^{23}$ to calculate the optimal values for passive shunt $\mathrm{L}_{\mathrm{opt}}=1 /\left(\mathrm{C}_{\mathrm{w}}{ }_{\mathrm{n}}{ }^{2}\right)$. In which $\mathrm{C}$ is the capacitance of the piezoelectric layer used and $\mathrm{w}_{\mathrm{n}}$ is the natural frequency at which vibration is to be decreased. With the natural frequency $\mathrm{w}_{\mathrm{n}}=869 \mathrm{~Hz}, \mathrm{~L}_{\text {opt }}=8.1 \mathrm{H}$. Because the inductance values are very high, they are not found commercially. Some inductors were manufactured and these were used in series to obtain the closest configuration to the numerically found, and the inductors used were: $\mathrm{L}=(6.19+0.51+0.38) \mathrm{H}=7.8 \mathrm{H}$. This variation of $3.7 \%$ can still provide a good vibration damping on the natural frequency, as shown by Venter and Silva ${ }^{24}$. The internal resistance of the inductors in series was measured at $350 \Omega$, so it was necessary to use an additional resistance in order to provide a good bandwidth for the passive shunt, and then $\mathrm{R}_{\text {total }}=1.55 \mathrm{k} \Omega$, approximated value which was obtained in a optimization simulation using the method provided by Hagood and von Flotow ${ }^{23}$.

Importantly, as the piezoelectric actuators used were on opposite sides of the tool-holder, the actuator terminals must be mounted on a protoboard keeping in mind the polarization direction. Thus, as one actuator expands, the other contracts, doubling the control action applied to the tool-holder and acting as a parallel actuator.

Open-loop, passive-controlled closed-loop and active-controlled closed-loop experiments were then performed to verify the feasibility of using serial multilayer piezoelectric actuators to increase the control capability of the piezoelectric actuators.

In the case of active control validation, the aforementioned active velocity feedback control scheme was used. The piezo amplifier used was the TREK PZD 700, which provides up to $700 \mathrm{~V}$ as output.

\section{Results and Discussion}

The experiments were performed under the two different fixation conditions shown in Figure 6. The experimental frequency response functions are presented for open circuit 


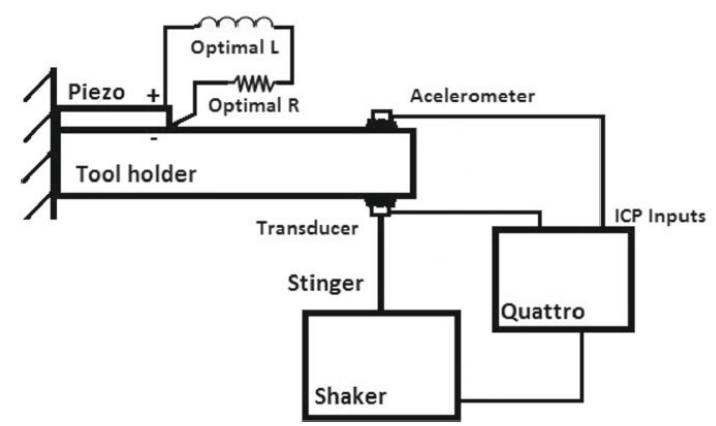

(a)

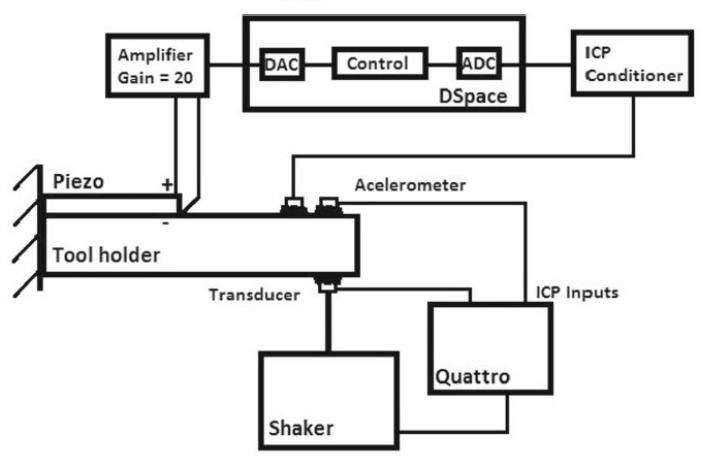

(b)

Figure 7. Schematic experimental setup a) passive control and b) active control, adapted from (18). and passive and active control cases in both fixations. The parameters of passive shunts used for passive control are the values described above.

The first experiment was performed in the first fixation configuration, where the tool-holder is carefully aligned with the reference system (Figure 6a). Passive shunt values are as described and several distinct gains for active control were tested. The gains used were: $\mathrm{K}_{1}=1160$, which guarantees a limit of approximately $180 \mathrm{~V}$ of electrical tension applied at the peak speed; $\mathrm{K}_{2}=2000$, which guarantees a limit of $300 \mathrm{~V}$ of electrical tension applied at the peak speed; and $\mathrm{K}_{3}=3200$, which guarantees a limit of $480 \mathrm{~V}$ of electrical tension at the peak speed. Figure 9 shows that both passive shunt and speed control can achieve a good reduction in vibration amplitude.

As can be seen in Figure 9, the tool-holder has a natural frequency $\mathrm{w}_{\mathrm{n}}=869 \mathrm{~Hz}$. At this frequency, the passive control was able to achieve a $1.3 \mathrm{~dB}$ reduction in the measured FRF amplitude. Active velocity feedback control was able to achieve reductions of up to $6.61 \mathrm{~dB}$ (range $62.79 \mathrm{~dB}$ to $69.4 \mathrm{~dB}$ ) when a maximum electric tension of $480 \mathrm{~V}$ was applied. In linear scale, these peaks represent $2864 \mathrm{~mm} / \mathrm{s}^{2} / \mathrm{N}$ for the open circuit and $1379 \mathrm{~mm} / \mathrm{s}^{2} / \mathrm{N}$ for the active control with $\mathrm{K}_{3}$. Thus, it is possible to see a drop of more than $50 \%$ in the vibration amplitude in the case of higher voltage applied to the multilayer piezoelectric actuator. This drop is much higher than those obtained with simple piezoelectric actuators, as seen in da Silva et al., 2015 and Venter et al., 2018, which present maximum reductions in vibration amplitude of about $20 \%$ and $40 \%$, respectively. In these papers it has been shown

\section{RTI Data}

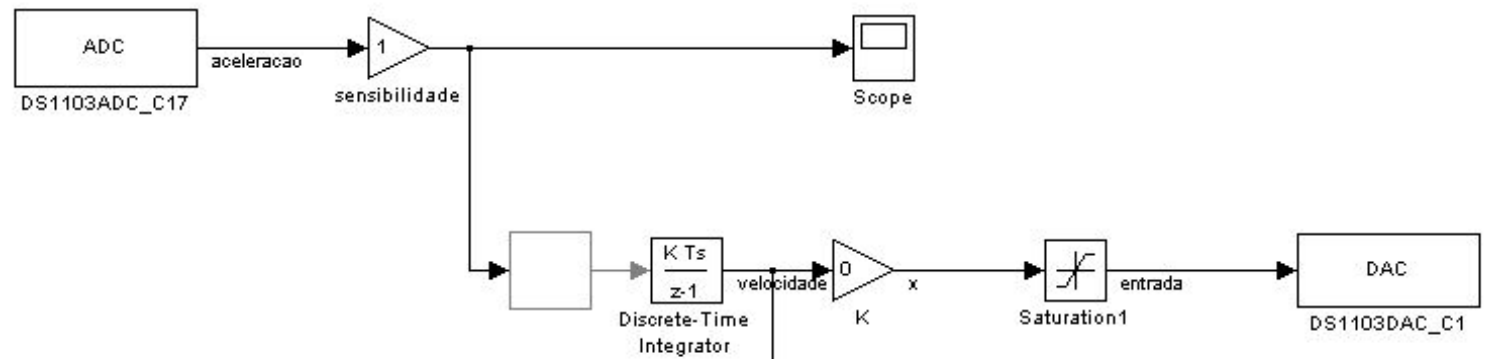

Figure 8. Simulink active control loop. 


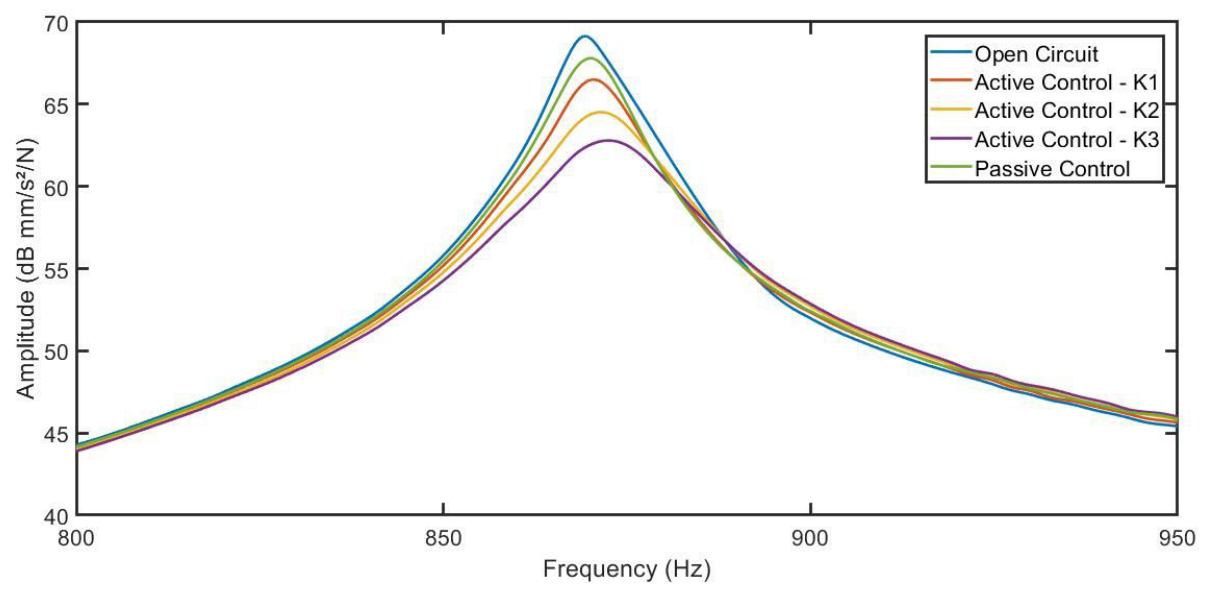

Figure 9. Experimental FRF amplitude with parallel multilayer piezoelectric actuator for first configuration.

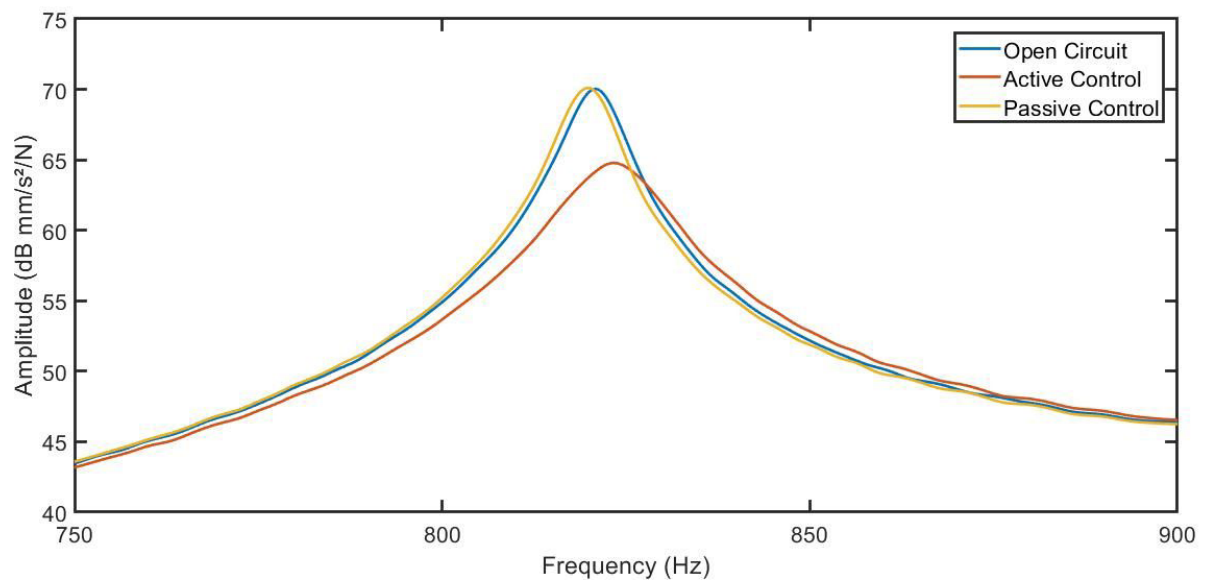

Figure 10. Experimental FRF amplitude with parallel multilayer piezoelectric actuator for second configuration.

Table 1. Polynomial multi-mode system identification parameters for the $1^{\text {st }}$ configuration.

\begin{tabular}{ccc}
\hline & $\mathrm{w}_{\mathrm{n}}[\mathrm{Hz}]$ & $\zeta$ \\
\hline Open Circuit & 875.7 & 0.0353 \\
\hline Passive strategy & 874.2 & 0.0358 \\
\hline Active strategy - K1 & 873.8 & 0.0364 \\
\hline Active strategy - K2 & 875.7 & 0.0425 \\
\hline Active strategy - K3 & 875.9 & 0.0481 \\
\hline
\end{tabular}

that this vibration drop results in an improvement in the roughness of the end piece, corroborating the result that this multilayer piezoelectric actuator can be applied with great possibilities for surface roughness improvement.

Using a polynomial multi-mode system identification, set for four-degrees-of-freedom in order to obtain the best approximation of the FRF peak, it is possible to obtain the data presented in Table 1. The data presented is regarding the system's first natural frequency, which can be seen in Figure 8 .

As can be seen in Table 1, the active strategy greatly improves the damping ratio of the system, presenting a gain of up to $36 \%$ in damping with the use of K3. Given that the system did not saturate using $\mathrm{K} 3$ as a gain in the active velocity feedback control strategy, damping improvement even higher could be obtained with higher applied electrical tensions. As seen in Section 2, the increase in the damping ratio directly changes the stability limit, thus the strategy might be suitable to control chatter vibration on machining.

For the second experiment, the tool-holder was intentionally misaligned, as shown in Figure 6b). The different fixation position resulted in a variation in the natural frequency of the structure, which would alter the passive shunt parameters. As passive shunt parameters were set for both experiments, differences should arise in the estimated FRF amplitude.

Figure 10 shows this difference. Passive control in this case could not decrease the vibration amplitude, given that the natural frequency of the structure changed to $\mathrm{w}_{\mathrm{n}}=821 \mathrm{~Hz}$. In this case, the optimal shunt values would have to be changed to obtain a reduction in the vibration amplitude. Using a gain of $\mathrm{K}=3200$ on the active control, which represents a limit of up to $480 \mathrm{~V}$ on peak vibration, gives the amplitudes of the FRF shown in Figure 9. As can be seen, active velocity control was able to increase the damping, achieving an approximately $5.3 \mathrm{~dB}$ reduction in the vibration amplitude.

Using a polynomial multi-mode system identification, set for four-degrees-of-freedom in order to obtain the best approximation of the FRF peak, it is possible to obtain the data presented in Table 2 . The passive case was not analyzed 
Table 2. Polynomial multi-mode system identification parameters for the $2^{\text {nd }}$ configuration.

\begin{tabular}{ccc}
\hline & $\mathrm{w}_{\mathrm{n}}[\mathrm{Hz}]$ & $\zeta$ \\
\hline Open Circuit & 823.0 & 0.0348 \\
\hline Active strategy & 822.8 & 0.0447 \\
\hline
\end{tabular}

being virtually the same curve as the open circuit FRF in Figure 10. The data presented is regarding the system's natural frequency, which can be seen in Figure 10.

As can be seen in Table 2, the active strategy still provides a great improvement in the damping ratio of the system, proving it to be a suitable and robust strategy. As seen in Section 2, the increase in the damping ratio directly changes the stability limit, thus the strategy is proven to be suitable to control chatter vibration on machining.

With this reduction in vibration amplitude, this multilayer piezoelectric actuator with the active control strategy can be used in operational applications to decrease vibration during the machining process. Further studies are needed in order to use microcontrollers and amplifiers available on the machine itself, rendering the active control solution feasible in real applications. In addition, further studies can be conducted to study the possibility of using energy harvesting to power the active control, eliminating the need for amplifiers.

\section{Conclusions}

A methodology to manufacture different multilayer piezoelectric actuators was presented. The actuators in series were tested in a controlled experimental setup to validate its ability to control vibration. This experimental campaign aimed to prove the feasibility of increasing the possible reduction in vibration amplitude using multilayer piezoelectric actuators when compared to single-layer piezoelectric actuators. The multilayer piezoelectric actuators were proven to be able to significantly reduce vibration amplitude, with a maximum of $36 \%$ increase in the structural damping and a $50 \%$ decrease in the vibration amplitude. The increase in the damping ratio directly changes the stability limit, thus the strategy is suitable to control chatter vibration on machining. The actuators remained robust when tested with robust control strategies (velocity feedback control). Therefore, a multilayer piezoelectric actuator can be a good choice for vibration control in internal turning, aiding in suppressing chatter.

\section{Acknowledgements}

This research was supported by grants CNPq 470314/2010-9, 479342/2012-1, FAPESP 2016/11309-0 and EMVeM ITN 315973. Moreover, Giuliana Sardi Venter is thankful for her grant FAPESP 2014/23108-4.

\section{References}

1. Wiercigroch M, Budak E. Sources of nonlinearities, chatter generation and suppression in metal cutting. Philosophical Transactions of the Royal Society A: Mathematical, Physical and Engineering Sciences. 1781;2001(359):663-93.

2. Muhammad BB, Wan M, Feng J, Zhang WH. Dynamic damping of machining vibration: a review. Int J Adv Manuf Technol. 2017;89(9-12):2935-52.
3. Wiercigroch M, Krivtsov AM. Frictional chatter in orthogonal metal cutting. Philosophical Transactions of the Royal Society A: Mathematical, Physical and Engineering Sciences. 1781;2001(359):713-38.

4. Ganguli A, Deraemaeker A, Preumont A. Regenerative chatter reduction by active damping control. J Sound Vibrat. 2007;300(3-5):847-62.

5. Yang Y, Zhang WH, Ma YC, Wan M. Chatter prediction for the peripheral milling of thin-walled workpieces with curved surfaces. Int J Mach Tools Manuf. 2016;109:36-48.

6. Quintana G, Ciurana J. Chatter in machining processes: A review. Int J Mach Tools Manuf. 2011;51(5):363-76.

7. Siddhpura M, Paurobally R. A review of chatter vibration research in turning. Int J Mach Tools Manuf. 2012;61:27-47.

8. Munoa J, Beudaert X, Dombovari Z, Altintas Y, Budak E, Brecher $\mathrm{C}$, et al. Chatter suppression techniques in metal cutting. CIRP Ann. 2016;65(2):785-808.

9. Tobias SA, Gurney JP. A graphical method for the determination of the dynamic stability of machine tools. International Journal of Machine Tool Design and Research. 1961;1:148-56.

10. Tlusty J, Polacek M. The stability of machine tools against self-excited vibrations in machining.. In Proceedings of the International Research in Production Engineering Conference; 1963 Sep 9-12; Pittsburgh, PA. Proceedings. New York: ASME; 1963. p. 465-74.

11. Merrit HE. Theory of self-excited machine-tool chattercontribution to machine tool chatter research-1. ASME Journal of Engineering for Industry. 1965;87(4):447-54.

12. da Silva MM, Cervelin JE, Arellano DPC, Coelho RT. Availability study on regenerative chatter avoidance in turning operations through passive and active damping. International Journal of Mechatronics and Manufacturing Systems. 2013;6:455-73.

13. Urbikain G, Fernández A, López de Lacalle LN, Gutiérrez ME. Stability lobes for general turning operations with slender tools in the tangential direction. Int J Mach Tools Manuf. 2013;67:35-44.

14. Liu Y, Li T, Liu K, Zhang Y. Chatter reliability prediction of turning process system with uncertainties. Mech Syst Signal Process. 2016;66-67:232-47.

15. Erturk A, Inman DJ. Piezoelectric shunt damping for chatter suppression in machining processes. In: Proceedings of the International Conference on Noise and Vibration Engineering; 2008; Leuven, Belgium. Porceedings. p. 193-206.

16. Ema S, Marui E. Suppression of chatter vibration of boring tools using impact dampers. Int J Mach Tools Manuf. 2000;40(8):1141-56.

17. da Silva MM, Venter GS, Varoto PSP, Coelho RT. Experimental results on chatter reduction in turning through embedded piezoelectric material and passive shunt circuits. Mechatronics. 2015;29:78-85.

18. Diniz AE, da Silva WTA, Suyama DI, Pederiva R, Albuquerque MV. Evaluating the use of a new type of impact damper for internal turning tool bar in deep holes. Int J Adv Manuf Technol. 2019;101(5-8):1375-90.

19. Viana FAC, Steffen V. Multimodal vibration damping through piezoelectric patches and optimal resonant shunt circuits. J Braz Soc Mech Sci Eng. 2006;28(3):293-310.

20. Venter GS, Coelho RT, Silva MM. Controle de vibração em porta-ferramenta de torneamento interno com atuador piezelétrico multicamadas. In: Anais do $10^{\circ}$ Congresso Brasileiro de Engenharia de Fabricação; 2019 Ago 5-7; São Carlos. Proceedings. São Carlos: UFSCar; 2019. p. 1-6.

21. Neugebauer R, Denkena B, Wegener K. Mechatronic systems for machine tools. CIRP Ann. 2007;56(2):657-86.

22. Venter GS. Silva LM do P, Carneiro MB, da Silva MM. Passive and active strategies using embedded piezoelectric layers to 
improve the stability limit in turning/boring operations. Int $\mathrm{J}$ Adv Manuf Technol. 2017;89(9-12):2789-801.

23. Hagood NW, von Flotow A. Damping of structural vibrations with piezoelectric materials and passive electrical networks. J Sound Vibrat. 1991;146(2):243-68.
24. Venter GS, Silva MM. On the robustness of the passive shunt control strategy for chatter reduction. In: Proceedings of the ISMA2016 International Conference on Noise and Vibration Engineering and USD2016 International Conference on Uncertainty in Structural Dynamics. 2016; Leuven, Belgium; p. 3721-3734. 